\title{
Dank den Referenten
}

Die Herausgeber und wohl auch fast alle Autoren bedanken sich sehr herzlich bei den Gutachtern, die einen Teil ihrer Arbeitskraft für das Zustandekommen der Beiträge in dieser Zeitschrift, so wie sie sind bzw. nicht sind, investiert haben. Während der Entstehungszeit von Band 17 haben referiert:

E. Aarts, Eindhoven

A. Agnetis, Rom

S. Albers, Kiel

M. Bastian, Aachen

J. Bendisch, St. Augustin

C. Bierwirth, Bremen

P. Borm, Tilburg

H.W. Brachinger, Fribourg

P. Brandimarte, Turin

F. Catthoor, Leuven

D. Cattrysse, Leuven

T.C. Cheng, Hong Kong

D. Chhajed, Urbana-Champaign

B.D. Choi, Seoul

J.J.M. Deerks, Maastricht

M. Dell'Amico, Mailand

U. Derigs, Köln

N. van Dijk, Amsterdam

W. Dinkelbach, Saarbrücken

U. Dorndorf, Aachen

K. Dowsland, Swansea

A. Drexl, Kiel

B. Fleischmann, Augsburg

B. Fox, Denver

A. Freville, Valenciennes

I. Garcia-Jurado, Santiago de Compostella

T. Gau, Braunschweig

W. Gaul, Karlsruhe

H.-O. Günther, Berlin

T. Hanschke, Clausthal

R. Haupt, Jena

K. van Hee, Eindhoven

J.E. Higgins, Berkeley

L. Hildebrandt, Berlin

M. Holler, Hamburg

R. Hübscher, Boulder

J. Hurink, Osnabrück

H. Isermann, Frankfurt/M.

J. Jahn, Erlangen

W. Junginger, Hamburg

B. Jurisch, Osnabrück

A.B. Kahng, Los Angeles

R. Klein, Darmstadt

A. Kolen, Maastricht

R. Kolisch, Kiel

H. Kopfer, Bremen
G. Krispin, Darmstadt

W. Kubiak, St. Johns

H. Kuhn, Köln

L. Kuntz, Mainz

J. Leung,Tucson

A. Löbel, Berlin

A. Lokketangen, Molde

F. Malucelli, Pisa

V. Maniezzo, Ispra

K. Mathias, Fort Collins

S. Meyer, Hamburg

E. Nowicki, Wroclaw

M. Oosten, Pittsburgh

I. Osman, Kent

M. Padberg, New York

F. Patrone, Genua

J.F. Pekny, West Lafayette

E. Pesch, Bonn

J. Piehler, Merseburg

M. Pirlot, Mons

C. Potts, Southampton

C. Reeves, Coventry

G. Reinelt, Heidelberg

C.C. Ribeiro, Rio de Janeiro

K. Richter, Frankfurt/O.

M. Rijal, New York

M. Salomon, Rotterdam

G. Scheithauer, Dresden

H.Y. Schenk-Mathes Frankfurt/M.

H. Schmalen, Passau

A. Scholl, Darmstadt

T. Siegel, Berlin

J. Skorin-Kapov, Stony Brook

C. Smutnicki, Wroclaw

F. Spieksma, Leiden

R. Storer, Bethlehem (USA)

S. Strahringer, Darmstadt

S. Subrahmanyam, Rutherford

U. Suhl, Berlin

S. Tijs, Tilburg

S. Voß, Braunschweig

M. Weber, Mannheim

A. Weintraub, Santiago

P. Widmayer, Zürich

D. Woodruff, Davis 